\title{
Experimental Results on Adiabatic Coupling Into SOI Photonic Crystal Coupled-Cavity Waveguides
}

\author{
P. Sanchis, J. Martí, W. Bogaerts, P. Dumon, D. Van Thourhout, and R. Baets
}

\begin{abstract}
The experimental implementation of an adiabatic coupling technique for efficient coupling between photonic crystal single-line defect waveguides and coupled-cavity waveguides is reported. The samples were fabricated in a silicon-on-insulator substrate using 248-nm-deep ultraviolet lithography. Experimental results are compared with three-dimensional (3-D) finite-difference time-domain simulations. Furthermore, the discrepancies between two-dimensional and 3-D simulation results are analyzed.
\end{abstract}

Index Terms-Coupled-cavity, photonic crystals (PhCs), silicon-on-insulator (SOI), waveguides.

\section{INTRODUCTION}

$\mathbf{T}$ HE DISPERSION features of coupled-cavity waveguides (CCWs) make this kind of photonic crystal $(\mathrm{PhC})$ waveguide a good choice to implement a large variety of functionalities, such as very compact optical delay lines or dispersion compensators [1], [2]. CCWs, also called coupled-resonator optical waveguides, are realized by introducing a chain of strongly confined point defects or cavities into the otherwise perfect PhC [3]. One key issue that needs to be addressed for enabling definitive widespread $\mathrm{CCW}$-based devices is an efficient coupling from an external medium [4]. An adiabatic coupling technique between single-line defect waveguides (SLWGs) and CCWs was recently proposed [5]. Furthermore, the dynamic performance was also investigated by analyzing the transmission of ultrashort pulses through the structure [6]. The coupling technique is based on gradually varying the radii of the spacing defects between cavities in order to match the SLWG and CCW modes. Thus, efficient light transmission from SLWGs into and out of a CCW of finite length is achieved. In this letter, the experimental implementation of this coupling technique by using an air-hole $\mathrm{PhC}$ structure fabricated in silicon-on-insulator (SOI) is reported. These are, to the best of our knowledge, the first experimental results aimed to improve the coupling efficiency into CCWs.

Manuscript received November 19, 2004; revised February 11, 2005. This work has been supported in part by the Spanish Ministry of Science and Technology under Grant TIC2002-01553 and by the Generalitat Valenciana. This work was carried out in part in the framework of the European IST-PICCO Project and the European IST-ePIXnet Network of Excellence. The work of P. Sanchis was supported by a grant from the Spanish Ministry of Education, Culture and Sport.

P. Sanchis and J. Martí are with the Nanophotonics Technology Center, Universidad Politécnica de Valencia, Valencia 46022, Spain (e-mail: pabsanki@ntc.upv.es; jmarti@dcom.upv.es).

W. Bogaerts, P. Dumon, D. Van Thourhout, and R. Baets are with the Department of Information Technology, Ghent University-IMEC, Ghent B-9000, Belgium (e-mail: Wim.Bogaerts@ugent.be; Pieter.Dumon@intec.UGent.be; driesvt@intec.ugent.be; roel.baets@intec.ugent.be).

Digital Object Identifier 10.1109/LPT.2005.846621

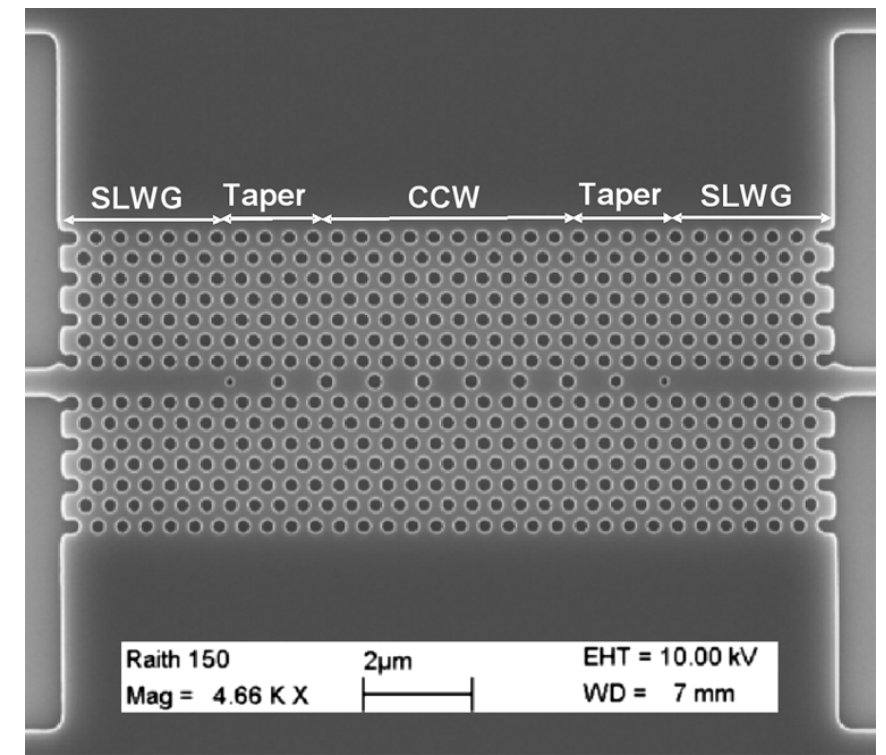

Fig. 1. Scanning electronic microscope (SEM) image of the CCW coupled to the SLWGs with the adiabatic taper. The taper is based on gradually varying the radii of the spacing holes between cavities.

\section{EXPERIMENTAL RESULTS}

The samples were fabricated on an 8 " SOI wafer using 248-nm-deep ultraviolet lithography [7], [8]. The SOI wafer consists of a 220-nm-thick silicon layer placed on top of a $1-\mu \mathrm{m}$-thick silica layer deposited over a bare silicon substrate. The $\mathrm{PhC}$ is formed by a two-dimensional (2-D) triangular array of air holes etched in the silicon layer. The lattice constant is $a=445 \mathrm{~nm}$ while the hole radius is $R=0.26 a$. The transmission as a function of wavelength was measured using an end-fire technique. Light from a tunable laser source was coupled into a 3- $\mu \mathrm{m}$-wide ridge waveguide using a lensed fiber. The output power of the laser was $1 \mathrm{~mW}(0 \mathrm{dBm})$. The samples consist of 3- $\mu \mathrm{m}$-wide ridge waveguides tapered down to a 500-nm-wide waveguides, best suited for coupling light into and out of the PhC. The output light was collected by an objective onto a power detector. A polarizer selecting the transverse-electric polarization and a diaphragm to collect only the output light from the ridge waveguide were used before the power detector.

CCWs of different lengths coupled to the SLWGs with and without taper were fabricated. Fig. 1 shows a scanning electronic microscope image of the $\mathrm{CCW}$ with taper structure. The adiabatic taper is formed by two intermediate holes. Experimental results have been compared with three-dimensional (3-D) finite-difference time-domain (FDTD) simulation results 

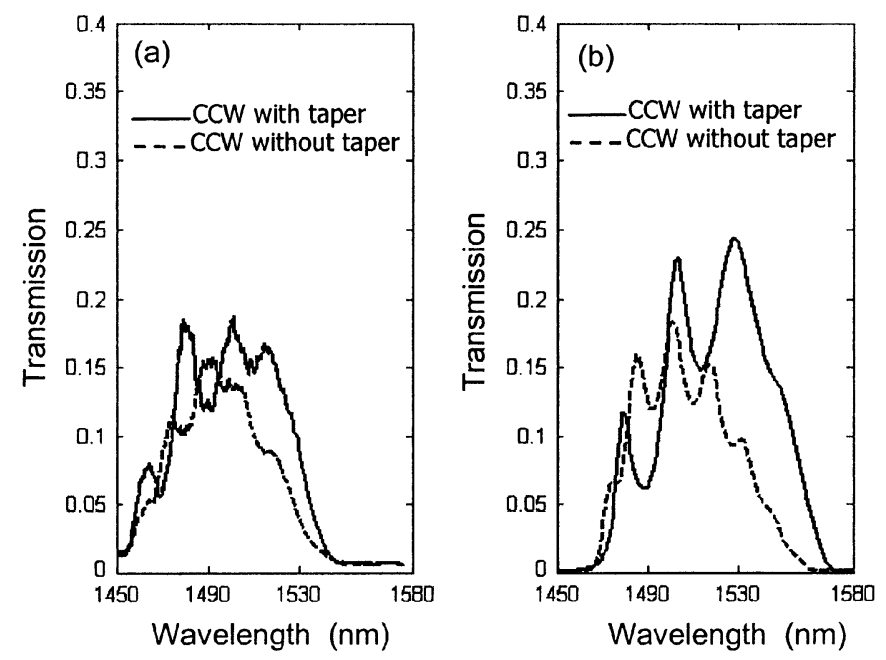

Fig. 2. (a) Experimental and (b) 3-D FDTD simulated transmission spectra as a function of the wavelength for a ten-cavities-long $\mathrm{CCW}$ and a nine-cavities-long taper/CCW/taper structure.
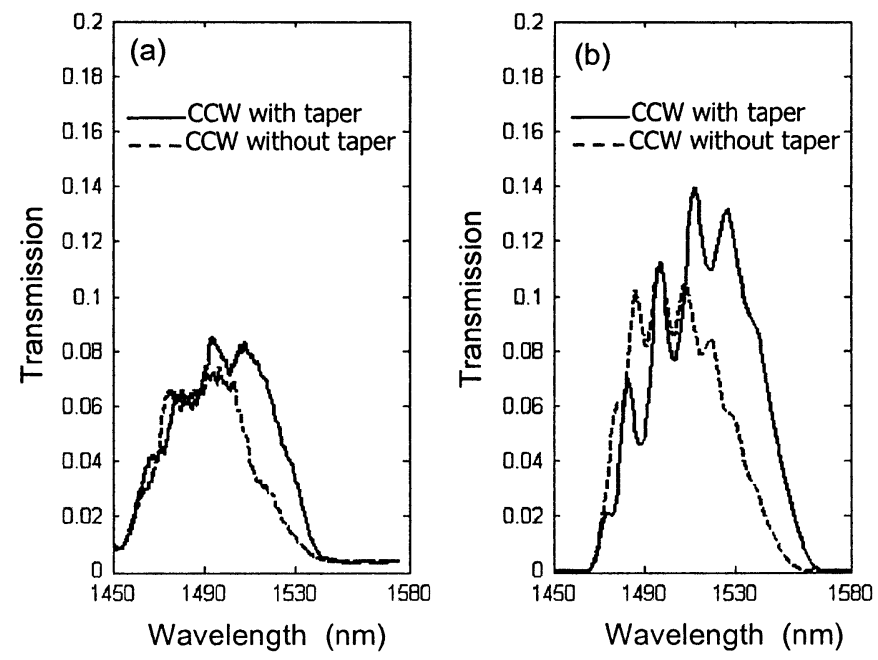

Fig. 3. (a) Experimental and (b) 3-D FDTD simulated transmission spectra as a function of the wavelength for a 15-cavities-long CCW and a 14-cavities-long taper/CCW/taper structure.

[9]. Fig. 2 shows the transmission efficiency as a function of the wavelength for a ten-cavities-long $\mathrm{CCW}$ and a nine-cavities-long taper/CCW/taper structure while Fig. 3 shows the transmission spectra for a 15-cavities-long CCW and a 14-cavities-long taper/CCW/taper structure. Experimental results have been normalized by the averaged transmission spectrum of the unpatterned structure. It can be seen that a very good agreement between experimental and 3-D FDTD results is obtained. The transmitted power is improved when the adiabatic taper, of only two intermediate holes, is used to couple light into and out of the CCW. However, transmission losses are still rather high. The only difference between the results shown in Figs. 2 and 3 is that the $\mathrm{CCW}$ has a different length since the taper length is not changed. Therefore, transmission losses are only increased in Fig. 3 due to the propagation losses in the CCW. Concretely, the transmitted power is decreased around $3 \mathrm{~dB}$ when the $\mathrm{CCW}$ length is increased by $4.45 \mu \mathrm{m}(10 a)$ so the propagation losses of the $\mathrm{CCW}$ are roughly estimated at $0.67 \mathrm{~dB} / \mu \mathrm{m}$. These rather high propagation losses are attributed to the out-of-plane losses
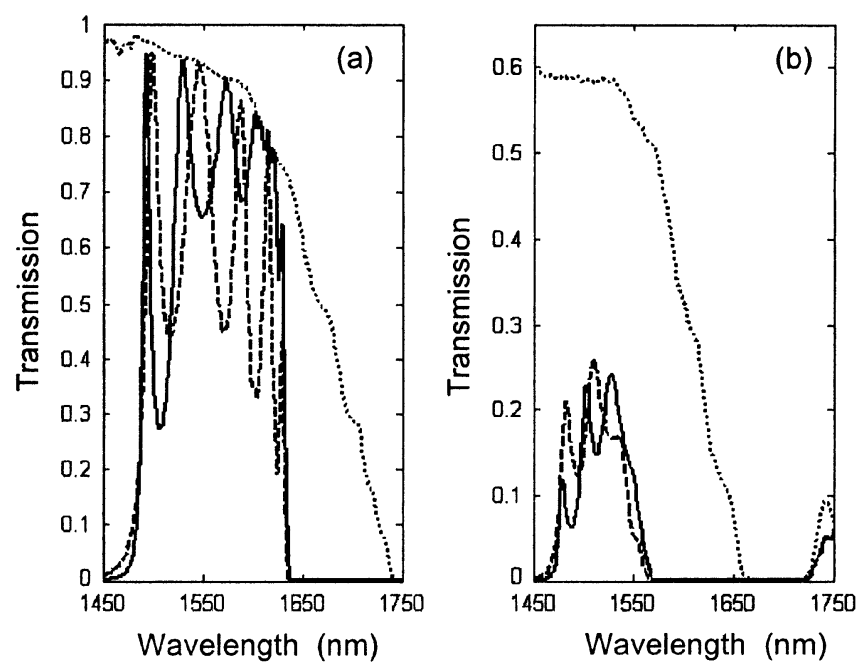

Fig. 4. (a) 2-D and (b) 3-D FDTD simulated transmission efficiency as a function of the wavelength for a five-cavities-long CCW coupled to the SWLGs without taper (dashed line) and with a taper formed by two intermediate holes (solid line). The dotted line depicts the transmission spectrum for a $12 a$-long SLWG, which corresponds to the total length of the input and output SLWGs used in the $\mathrm{CCW}$ with taper structure.

originated from the fact that the $\mathrm{CCW}$ operates above the light line, which implies that the guided mode is intrinsically lossy. It is important to remark that propagation losses due to sidewall roughness are not taken into account in the $3-\mathrm{D}$ simulations.

The transmission losses due to the estimated propagation losses are of $6.3 \mathrm{~dB}$ (23\% transmission) for a ten-cavities-long $\mathrm{CCW}$ and of $9.3 \mathrm{~dB}(11.75 \%$ transmission $)$ for a 15 -cavitieslong CCW. These values are close to those obtained for the CCW without taper structure, depicted as dashed lines in Figs. 2 and 3, which means that the propagation losses of the $\mathrm{CCW}$ are clearly dominant in the overall transmission losses. However, the transmission is higher for the $\mathrm{CCW}$ with taper structure, especially at the high wavelengths of the band. This transmission improvement is attributed to the effect of the taper but also because the $\mathrm{CCW}$ length is actually shorter in the $\mathrm{CCW}$ with taper structure since the total number of cavities including the input and output tapers is almost the same to that of the CCW without taper structure.

\section{DISCUSSION}

In order to better analyze the taper performance, the transmission spectra for a $\mathrm{CCW}$ of the same length coupled to the SLWG with and without the adiabatic taper have been calculated by means of 3-D FDTD simulations. The PhC has also the same length in both structures so the adiabatic taper has been replaced by an SLWG in the CCW without taper structure. Furthermore, 3-D simulation results have been compared to 2-D simulation results. In the latter, the effective index approximation has been used. The effective index $\left(n_{\text {eff }}=2.8\right)$ was calculated taking into account the thickness of the silicon layer of the fabricated structure. Fig. 4 shows the transmission spectra calculated by means of (a) 2-D and (b) 3-D FDTD simulations for a five-cavities-long CCW coupled to the SWLGs without taper (dashed line) and with a taper formed by two intermediate holes (solid line). On the other hand, Fig. 5 shows the 

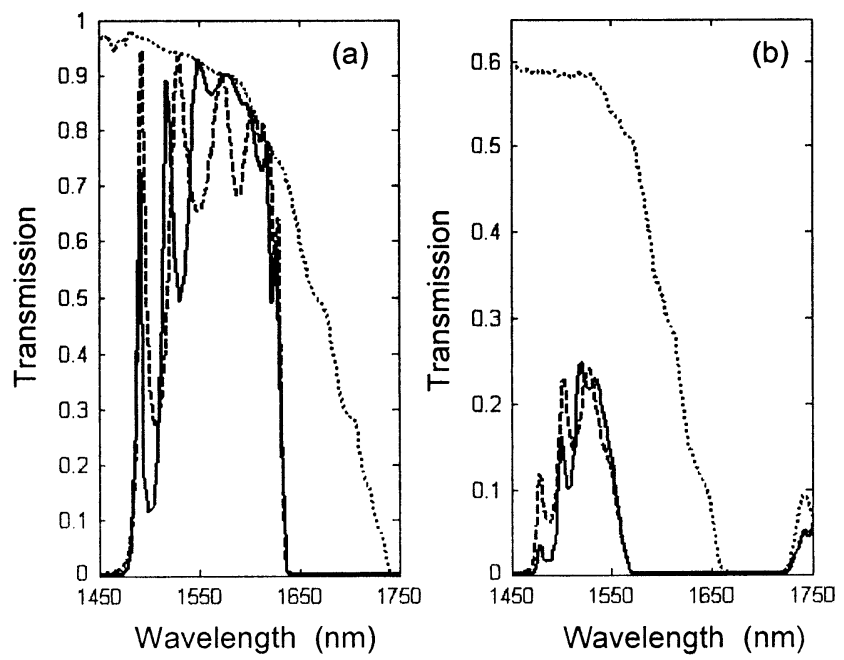

Fig. 5. (a) 2-D and (b) 3-D FDTD simulated transmission efficiency as a function of the wavelength for a five-cavities-long CCW coupled to the SWLGs with a taper formed by two (dashed line) and four (solid line) intermediate holes. The dotted line depicts the transmission spectrum for a $12 a$-long SLWG.

2-D and 3-D transmission spectra for a five-cavities-long CCW coupled to the SWLGs with a taper formed by two (dashed line) and four (solid line) intermediate holes. In both Figs. 4 and 5, the transmission spectrum is also shown for a $12 a$-long SLWG (dotted line), which corresponds to the total length of the input and output SLWGs used in the CCW with taper structure considering a taper length of two intermediate rows.

When no taper is used, a number of resonances peaks equal to the number of cavities appear in the 2-D simulated transmission spectrum, as it can be seen in Fig. 4(a). The peak-to-valley ratio of the undesired resonance peaks are reduced when the adiabatic taper is inserted into the structure. The improvement occurs at the high wavelengths of the transmission band while sharper resonant peaks appear at the low wavelengths of the band. The latter is originated due to the violation of the condition of adiabaticity occurred for those wavelengths at intermediate points in the taper [5]. However, the wavelength range, in which a rather flat transmission is observed, grows as the taper length increases, as it can be seen in Fig. 5(a).

The transmission improvement provided by the taper is not so evident for the 3-D results shown in Figs. 4(b) and 5(b). However, this is not due to a bad performance of the taper. In Figs. 4(b) and 5(b), it can be seen that the transmission efficiency decreases in the $12 a$-long SLWG structure as the wavelength is closer to the band edge due to the larger coupling losses between the external dielectric waveguide and the SLWG. Furthermore, the cutoff wavelength is shorter than the one given for the 2-D results. These higher coupling losses besides the high propagation losses in the $\mathrm{CCW}$ make negligible the transmission efficiency at the high wavelengths of the $\mathrm{CCW}$ band, which restricts the transmission improvement provided by the taper. The transmission efficiency is also attenuated at higher wavelengths in the 2-D results, as depicted in Figs. 4(a) and 5(a). However, it is much less critical due to the lack of out-of-plane losses. Thus, the 3-D simulated transmission efficiency is improved at higher wavelengths while it is worse at lower wavelengths due to the effect of the taper, which agrees with the results predicted by the 2-D results. This behavior is enhanced as the taper length increases, as it can be seen in Fig. 5(b). Furthermore, the transmission improvement due to the taper explains why the cutoff wavelength of the upper band edge of the $\mathrm{CCW}$ with taper structure is above that of the CCW without taper structure while the cutoff wavelength of the lower band edge is the same in both structures, as it can be seen in Figs. 2(b), 3(b), or 4(b).

The previous analysis confirms that the transmission improvement shown at higher wavelengths in the experimental results for the $\mathrm{CCW}$ with taper structure (see Figs. 2 or 3 ) is attributed to the higher coupling efficiency between the SLWG and CCW. However, the overall transmission losses are lower because the propagation losses in the taper are lower than the propagation losses in the $\mathrm{CCW}$.

\section{CONCLUSION}

Experimental results of an adiabatic coupling technique to improve the coupling efficiency into CCWs have been reported and compared with 2-D and 3-D FDTD simulation results. The results show that out-of-plane losses are rather high in the $\mathrm{CCW}$ significantly increasing propagation losses and restricting the transmission improvement provided by the taper. The analysis of out-of-plane losses and techniques to avoid them will become necessary to improve the $\mathrm{CCW}$ performance.

\section{REFERENCES}

[1] S. Lan, S. Nishikawa, H. Ishikawa, and O. Wada, "Design of impurity band-based photonic crystal waveguides and delay lines for ultrashort optical pulses," J. Appl. Phys., vol. 90, pp. 4321-4327, 2001.

[2] T. J. Karle, Y. J. Chai, C. N. Morgan, I. H. White, and T. F. Krauss, "Observation of pulse compression in photonic crystal coupled cavity waveguides," J. Lightw. Technol., vol. 22, no. 2, pp. 514-519, Feb. 2004.

[3] A. Yariv, Y. Xu, R. K. Lee, and A. Scherer, "Coupled resonator optical waveguides: A proposal and analysis," Opt. Lett., vol. 24, pp. 711-713, 1999.

[4] T. J. Karle, D. H. Brown, R. Wilson, M. Steer, and T. F. Krauss, "Planar photonic crystal coupled cavity waveguides," IEEE J. Sel. Topics Quantum Electron., vol. 8, no. 4, pp. 909-918, Jul./Aug. 2002.

[5] P. Sanchis, J. Garcia, A. Martínez, F. Cuesta, A. Griol, and J. Martí, "Analysis of adiabatic coupling between photonic crystal single-linedefect and coupled-resonator optical waveguides," Opt. Lett., vol. 28 , pp. 1903-1905, 2003.

[6] P. Sanchis, J. García, A. Martinez, and J. Martí, "Pulse propagation in adiabatically coupled photonic crystal coupled cavity waveguides," $J$. Appl. Phys., vol. 97, pp. 013 101-1-013 101-9, 2005.

[7] W. Bogaerts, D. Taillaert, B. Luyssaert, P. Dumon, J. Van Campenhout, P. Bienstman, D. Van Thourhout, R. Baets, V. Wiaux, and S. Beckx, "Basic structures for photonic integrated circuits in Silicon-on-insulator," Opt. Express, vol. 12, pp. 1583-1591, 2004.

[8] W. Bogaerts, R. Baets, P. Dumon, V. Wiaux, S. Beckx, D. Taillaert, B. Luyssaert, J. Van Campenhout, P. Bienstman, and D. Van Thourhout, "Nanophotonic waveguides in silicon-on-insulator fabricated with CMOS technology," J. Lightw. Technol. (invited), vol. 23, no. 1, pp. 401-412, Jan. 2005.

[9] A. Taflove, Computational Electrodynamics: The Finite-Difference Time-Domain Method. Norwood, MA: Artech, 1995. 